La revue La revue pour l'histoire du CNRS

POUR LHISTORE DU CNRS $\quad 24 \mid 2009$

Soixante-dixième anniversaire du CNRS

\title{
Un jour, la Chine s'est réveillée
}

Jean-Philippe Béja

\section{OpenEdition}

Journals

Édition électronique

URL : https://journals.openedition.org/histoire-cnrs/9155

DOI : 10.4000/histoire-cnrs.9155

ISSN : 1955-2408

Éditeur

CNRS Éditions

Édition imprimée

Date de publication : 5 octobre 2009

ISSN : 1298-9800

Référence électronique

Jean-Philippe Béja, «Un jour, la Chine s'est réveillée », La revue pour l'histoire du CNRS [En ligne], 24 I

2009, mis en ligne le 05 octobre 2009, consulté le 20 mai 2021. URL : http://journals.openedition.org/ histoire-cnrs/9155; DOI : https://doi.org/10.4000/histoire-cnrs.9155

Ce document a été généré automatiquement le 20 mai 2021.

Comité pour l'histoire du CNRS 


\title{
Un jour, la Chine s'est réveillée
}

\author{
Jean-Philippe Béja
}

1 En décembre 2008, le Parti communiste chinois a fêté le trentième anniversaire de l'adoption de la politique de réforme et d'ouverture. Si au cours de la dernière décennie, hommes d'affaires, enseignants, chercheurs ont pu se rendre facilement en Chine pour y enseigner, faire du commerce, ou participer à des colloques, la situation était loin d'être aussi idyllique à mes débuts en 1975.

2 Je me trouvais en effet face à un cruel dilemme: accepter une bourse d'études en République populaire de Chine ou intégrer un laboratoire du CNRS qui venait tout juste de me recruter. Tout tremblant, j'allai informer le directeur de mon laboratoire, le Ceri, que j'étais naturellement prêt à abandonner cette bourse pour prendre mes fonctions. Sentit-il mes regrets? Sa réponse fut catégorique : «Vous n’y pensez pas! Vous serez l'un des rares chercheurs en sciences sociales à pouvoir se rendre sur le terrain, et même si vous ne pouvez travailler directement sur votre programme de recherche, il s'agit d'une occasion inespérée. Vous serez bien plus utile en Chine que Boulevard Raspail !»

3 Et c'est ainsi que j'ai pu, au cours de cette année d'étude, me familiariser avec le fonctionnement de la bureaucratie chinoise, étudier directement la « révolution dans l'éducation " qui faisait rage, et accumuler une expérience qui est encore aujourd'hui inestimable car elle me permet d'établir une complicité avec les membres de la génération des Gardes rouges qui jouent actuellement un rôle central dans la vie de la République populaire de Chine.

4 Quelle autre institution que le CNRS aurait accepté d'entretenir un chercheur pour acquérir une telle formation hautement difficile à évaluer? Et pourtant, cette familiarité avec le fonctionnement du système et cette expérience directe de la vie dans la dernière décennie du règne de Mao sont des atouts inestimables pour un politologue. Au cours des années 1980 et jusqu'au milieu des années 1990, les politologues étrangers travaillant sur la Chine n'étaient pas accueillis avec enthousiasme par les autorités. Alors que mes collègues spécialistes de la France ou des relations internationales pouvaient se rendre aisément à l'Académie des sciences sociales de Chine dans le cadre des accords signés par celle-ci avec le CNRS, il n'en allait pas de même des « sinologues ». C'était encore plus difficile pour moi, étant donné que je travaille sur les 
relations entre le citoyen et le pouvoir, sur l'intelligentsia et le mouvement pour la démocratie.

Inutile de dire que jamais une mission sur ces thèmes n'aurait été accueillie par les institutions chinoises d'enseignement et de recherche. Il fallait donc partir comme touriste, sans invitation. Ce qui ne m'empêchait pas de rencontrer mes collègues, ni d'aller enquêter sur le terrain grâce à mes guanxi, ces relations personnelles qui sont si importantes en Chine. Contrairement à ce que je pensais, il a été relativement facile de convaincre mes autorités de tutelle que ces missions informelles étaient bien plus utiles que les missions officielles au cours desquelles il était extrêmement difficile d'échapper aux attentions du bureau des affaires étrangères de l'Académie des sciences sociales de Chine ou des universités. Là encore, où peut-on trouver une institution comprenant les contraintes qui pèsent sur les chercheurs?

6 Au milieu des années 1990, le CNRS m'a permis d'occuper le poste de directeur scientifique du Centre d'études français sur la Chine contemporaine. Dans ce centre, qui publie une revue très appréciée dans le milieu des études chinoises, Perspectives chinoises, j'ai pu à la fois poursuivre mes recherches sur les forces démocratiques en République populaire et mes travaux sur l'émergence d'une communauté politique à Hong Kong. Mais nous avons également pu donner l'occasion à des doctorants de se rendre sur le terrain pour de longues missions, et de former ainsi des chercheurs qui connaissent bien les institutions, la société et les forces politiques chinoises. Nous avons initié et mis en place des coopérations intenses avec les établissements de recherche et les universités chinoises et hongkongaises. Ce centre, financé par le ministère des Affaires étrangères et le CNRS nous est envié par tous nos collègues étrangers.

7 Pour un chercheur, il est très important de disposer de temps, d'emprunter des chemins de traverse pour comprendre les facteurs qui déterminent les évolutions d'un système politique ou d'une société. Dans un pays comme la République populaire de Chine, dirigé par un parti unique qui cherche à empêcher les étrangers de mener des enquêtes indépendantes, il faut beaucoup de temps pour établir les réseaux de relations indispensables à la mise en œuvre d'enquêtes sérieuses. Comme ce sont des chercheurs qui évaluent nos travaux, ils sont conscients de l'importance de l'investissement en temps et en énergie qu'un chercheur doit effectuer pour pouvoir mener des recherches de qualité.

8 Parfois, il faut beaucoup de temps avant de publier l'article ou l'ouvrage qui viendra couronner ce travail. Le chercheur immergé dans un régime autoritaire ou, pis encore, post-totalitaire, est parfois obligé de se retenir de publier des travaux pour protéger ses sources. C'est notamment le cas lorsque l'on travaille sur des sujets aussi sensibles que l'opposition. À certains moments de l'histoire, lorsque les médias, les hommes politiques se trouvent confrontés à la nécessité de comprendre l'évolution de la Chine, ils lisent nos travaux, nous interrogent. C'est là qu'ils se rendent compte de l'utilité d'un établissement comme le CNRS et de l'apport si nécessaire de cette recherche fondamentale en sciences humaines et sociales. 


\section{ANNEXES}

\section{RÉSUMÉS}

Pour tout chercheur travaillant sur la Chine contemporaine, la question principale est celle de l'accès au terrain. Jean-Philippe Béja nous conte en véritable pionnier les "tribulations » d'un chercheur du CNRS en Chine.

\section{AUTEUR}

\section{JEAN-PHILIPPE BÉJA}

Jean-Philippe Béja est directeur de recherche au Centre d'études et de recherches internationales (Ceri). Il est actuellement mis à disposition du CEFC de Hong Kong. 